\title{
Senu Banl'z
}

\section{attgemählte Merke.}

3 weite $\mathfrak{A} \mathfrak{u}$ gabe.

Sunf 3 bunter ginnd.

\author{
$\mathfrak{B e r l i n}$. \\ Berlag von (G. Reimer. \\ 1865.
}





\section{Jean $\mathfrak{F} \mathfrak{a}$ l' \\ $\mathfrak{a} \mathfrak{H} \mathfrak{g} \mathfrak{g} \mathfrak{w} \mathfrak{a} \mathfrak{h} l t \mathfrak{e} \mathfrak{W}_{\mathfrak{e r}} \mathfrak{k} \mathfrak{k}$.}

Sunf 3 ehnter Band. 



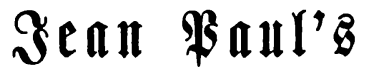

\section{auggewäl)lte Merke.}

3weite $\mathfrak{A} \mathfrak{u} \mathfrak{g a b e}$.

Suntzehnter Gand.

$\mathfrak{B} \operatorname{er} \mathfrak{I} \mathfrak{n}$.

Berlag von Bs. Reimer.

1865. 
